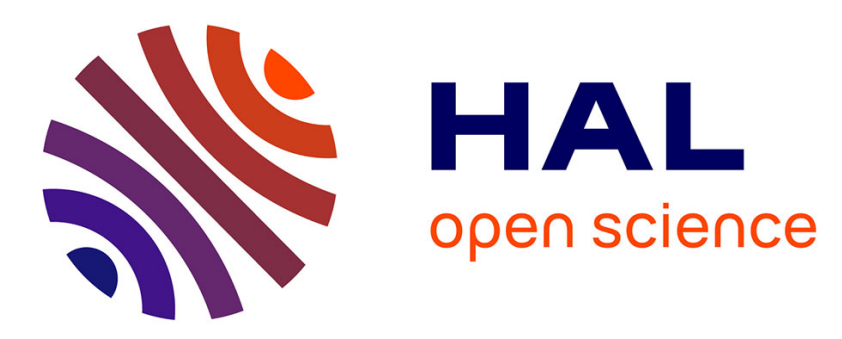

\title{
Global System Evaluation Scheme for Multiple Antennas Adaptive Receivers
}

Pierre-Francois Morlat, Herve Parvery, Guillaume Villemaud, Jacques Verdier, Jean-Marie Gorce, X. Xin

\section{- To cite this version:}

Pierre-Francois Morlat, Herve Parvery, Guillaume Villemaud, Jacques Verdier, Jean-Marie Gorce, et al.. Global System Evaluation Scheme for Multiple Antennas Adaptive Receivers. The 9th European Conference on Wireless Technology (EWCT), IEEE, Sep 2006, Manchester, United Kingdom. pp.197200, 10.1109/ECWT.2006.280469 . inria-00412076

\section{HAL Id: inria-00412076 \\ https://hal.inria.fr/inria-00412076}

Submitted on 27 Nov 2009

HAL is a multi-disciplinary open access archive for the deposit and dissemination of scientific research documents, whether they are published or not. The documents may come from teaching and research institutions in France or abroad, or from public or private research centers.
L'archive ouverte pluridisciplinaire HAL, est destinée au dépôt et à la diffusion de documents scientifiques de niveau recherche, publiés ou non, émanant des établissements d'enseignement et de recherche français ou étrangers, des laboratoires publics ou privés. 


\title{
Global System Evaluation Scheme for Multiple Antennas Adaptive Receivers
}

\author{
P. F. Morlat*, H. Parvery*, G. Villemaud*, X. Xin**, J. Verdier**, J. M. Gorce* \\ * CITI Laboratory - INSA Lyon, Lyon, France - ** LPM Laboratory - INSA Lyon, Lyon, France
}

\begin{abstract}
This paper describes a global system approach to easily develop, simulate and validate a multi-antennas receiver structure. Our aim is to offer a rapid evaluation for future wireless systems, including promising techniques such as SIMO or MIMO, Software Defined Radio (SDR), OFDM and Interference Cancellation. A particular focus is on the impact of RF front-ends characteristics on the effective performances of a receiver in a realistic environment. A complete connected solution based on Agilent Technologies tools is presented, combining simulations and measurements with realistic conditions. This testbed allows a direct evaluation of all parts of a wireless link with multiple antennas. For instance IQ imbalance and phase noise influence on a four antennas $802.11 \mathrm{~g}$ receiver is herein exposed.
\end{abstract}

Index Terms - Adaptive arrays, System analysis and design, Phase noise, MIMO systems.

\section{INTRODUCTION}

In the field of high data rate wireless systems, a very promising issue is to combine the most recent techniques in the same architecture dealing with numerous communication standards. For instance, this combination could be an OFDM signal associated with multiple antennas reconfigurable transceivers with a Zero-IF Software Defined Radio (SDR) structure [1].

In order to properly design such a transmission scheme, a complete and precise study based on both simulations and measurements is essential. This works aims to propose such a system-level evaluation by the use of a $2 \times 2$ MIMO radio platform connected to the Advanced Design System (ADS) software from Agilent Technologies [2]. This connected solution allows us to simulate and measure all parts of a wireless link, from the global system level down to the particular model of RF components or fading channels.

In part II, we discuss the key points of RF front-end limitations which strongly decrease receiver efficiency. Part III presents the system-level evaluation tools used in part IV describing a particular example of a 4 antennas $802.11 \mathrm{~g}$ receiver. In this example, we show some interesting results for the joint compensation of fading effects and IQ imbalance. Finally, the influence of the power amplifier nonlinearity and oscillator phase noise is reported in this paper.

\section{ON MULTIPLE ANTENNA RECONFIGURABLE RECEIVER PROBLEMS}

To properly balance subsystem performance, the digital signal processing engineer must be aware of the limitations of the analog RF front-end so that some compensation can be made via digital signal processing [4]. In most systems, the receiver tends to be more complex than the transmitter. The main challenge for designing receivers is the Dynamic Range. The RF front-end of a receiver must separate a desired signal typically from $-130 \mathrm{dBm}$ to $-70 \mathrm{dBm}$, from a background $\mathrm{RF}$ environment that may be in the relative range of $-20 \mathrm{~dB}$ up to $0 \mathrm{~dB}$. In many systems, the RF front-end also sets the system signal to noise ratio and should be designed to add minimal noise. Thus the overall system must have a considerable dynamic range to accommodate both the high-power background signals and the lowest-power desired signal. Dynamic range is limited at the bottom of the range by noise that enters the system through thermal effects of the components or through non-idealities of the ADC, such as quantization noise or sampling aperture jitter. Low level signals can be masked by this noise. Dynamic range is limited at the high-end by interference. The source of this interference could be co-channel, adjacent channel, or self induced by the transceiver. High levels of interference may cause the receiver to become more non-linear and introduce cross products (spurious components), which may inhibit the detection of low-level signals or reduce the desired signal bit error rate (BER). Simply attenuating the high-level signal before they drive the receiver into a non-linear operating region is insufficient since low-level desired signals also present will be attenuated until masked by system induced noise and thus will fall below the sensitivity of the receiver.

For the transmitter part, power-efficiency is a particularly challenging problem: power is consumed at a much higher level in the talk mode than in the stand-by mode. For a mobile unit, power control is commonly used at the mobile transmitter to reduce power consumption and to help improve capacity of the cellular system. The accuracy and linearity of the RF transmit chain is important to ensure that the desired signal is transmitted with no spurious signals or noise. Finally, noise and distortion are the limiting factor in the RF circuit performance, thus it is necessary to quantify them. Generally, several items are discussed in detail such as the noise figure of the receiver, the transmitter's noise floor, IQ gain imbalance, IQ orthogonality phase imbalance, phase noise, voltage noise of power supply, linear phase and amplitude distortion. Therefore, in a global system approach, both transmitter and receiver RF performances will impact the overall link budget. For instance, in the field of emerging high rate wireless systems, OFDM is a very popular modulation technique because of its performances in hard multi-path environments. Moreover Wireless LAN generally deal with 
such Rice or Rayleigh fading channels and so multiple antennas techniques appear an essential way of increasing throughput. At the same time, considerable effort is being made to develop reconfigurable receivers using more or less Software Defined Radio (SDR) principles. Thus multiple standards could elegantly cohabit in the same simple architecture with good processing capabilities.

Then a global approach combining multiple antennas, OFDM technique and SDR principles appears really promising particularly for future broadband wireless systems. Unfortunately, this combination is not so evident: multiple antenna algorithms and SDR are resource-expensive and OFDM technique is sensitive to RF front-end performances. Moreover, the impact of RF impairments on a receiver depends on RF front end topologies [5]. The direct conversion receivers often referred to as zero intermediate-frequency (Zero-IF) receivers have become very popular in wireless mobiles. Nevertheless, the RF impairments are much more critical in this case, as compared to super-heterodyne receivers for instance. In addition, a potential method is to sample a much larger bandwidth to deal with efficient interference cancellation or multi-channel capabilities. By the way, trying to enlarge the acquired bandwidth naturally degrades the RF performances.

\section{GLOBAL SySTEM EVALUATION APPROACH}

The radiocom platform (Fig. 1) is an analysis tool which allows arranging the most advanced techniques in conception, modelling and test for radiocommunication systems. This platform is made of high technology equipments developed by Agilent Technologies: the ADS software, and measurement hardwares which are two arbitrary waveform generators (ESG $4438 \mathrm{C}$ ) and a vector spectrum analyzer (VSA89641) with two RF inputs [2]. With this platform it is possible to extend measurements up to $6 \mathrm{GHz}$, with a received bandwidth analysis of $36 \mathrm{MHz}$. The arbitrary waveform generator by itself is able to generate any complex signal which it is then possible to analyze after propagation with the vector spectrum analyzer. The vectorial analysis software can demodulate this signal and offers visualisation options to show spectrum, constellations, BER, EVM... in order to accurately estimate the transmission system quality.

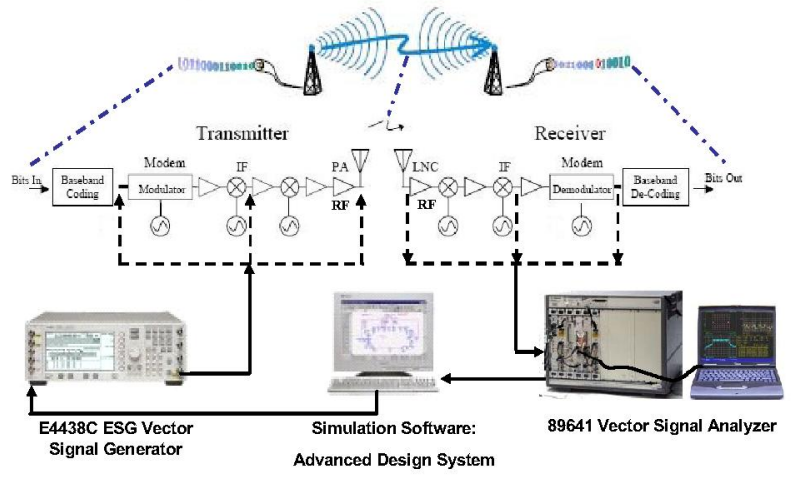

Fig. 1. The platform structure for a $1 \mathrm{x} 1$ transmission

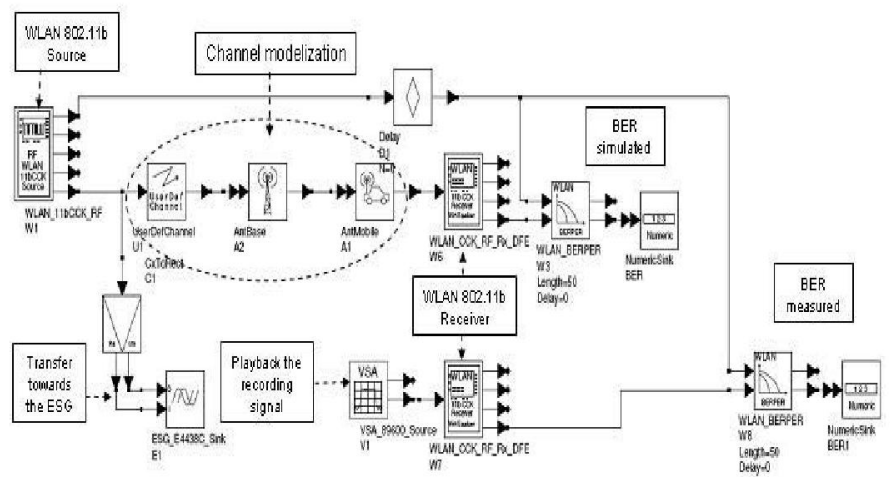

Fig. 2. Example of a schematic design for 802.11 b BER evaluation

With this connected solution a software/hardware interaction allows us to test and conceive very complex and realistic systems. Indeed the modelled signal with ADS can be loaded in the internal memory of the ESG, then the signal measured by the VSA can be recorded and transferred to the software. This interaction can take place at any part of the chain (baseband, RF...). ADS permits us to model the complete system, from the component to the whole architecture. With the Ptolemy tool, analogical and digital signal live together, it allows us to study all the fields bound to telecommunications by making them act mutually.

We can therefore estimate the impact of the different noise sources of the RF front end (phase noise, distortion, IQ imbalance...), and also the propagation environment (fading...). All component toolboxes (receiver, transmitter, channel) are customizable and models more or less elaborated can be add to them. A very realistic modelling of the system can be simulated taking into account the characteristic of the channel, and the physical properties of the front end. Fig. 2 presents an ADS schematic which evaluate the simulated and measured BER performance of an 802.11 b system versus the SNR.

For example, a part of the standard receiver and transmitter can be replaced by a specific component (VCO, LNA...) with their appropriate characteristics to evaluate their performance (Fig. 3). Finally, this platform permits the validation of channel models with direct comparison with signal recorded by the VSA in any typical environment. With the twice RF input of the VSA it is also possible to analyze and evaluate the performance of SIMO or MIMO systems or to characterize multi-channel models.

\section{EXAMPLE OF A FOUR ANTENNAS 802.11G RECEIVER WITH RF IMPAIRMENTS}

In this part, we will show via quantitative analysis that the SIMO configuration also helps to reduce the impact of nonideal characteristics of the RF front-end on OFDM receivers alongside mitigating the effects of fading. 


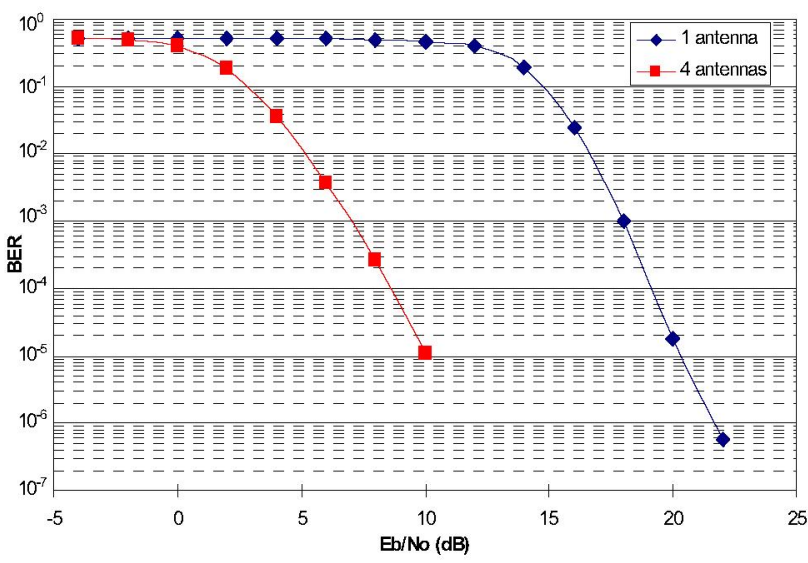

Fig. 4. BER vs. Eb/No in multipath channel

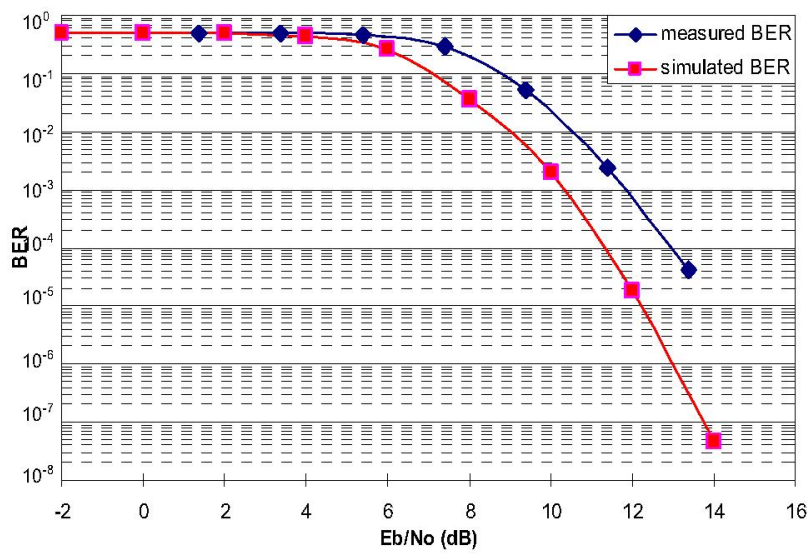

Fig. 5. BER vs. Eb/No in AWGN channel

Our objective is to simulate a very realistic transmission system and to have a better estimation of the advantage of the Sample Matrix Inversion (SMI) smart antennas algorithm [3]. A complete $802.11 \mathrm{~g}$ transmission system was modelled with the ADS software, using different types of channel (average white Gaussian noise or various multipath models). Fig. 4 presents the BER obtained with the four arms SMI receiver compared to a single antenna receiver with a $36 \mathrm{Mbps}$ transmission rate (16QAM). Fig. 5 compares simulated and measured BER performance.

\section{A. IQ Imbalance in AWGN and Multipath Channel}

Multiple antennas techniques are known to be efficient for signal-to-noise ratio improvement or interference rejection. By the way, RF impairments such as IQ imbalance could be considered as additional interference [4]. So minimisation techniques used for smart antennas applied on digital baseband signal allow a global compensation of channel effects and RF non-idealities. Thus a first evaluation of the SMI gain is presented in figures 6 and 7.

These results present the relative BER (zero gain imbalance BER value taken for reference) compared for a single antenna

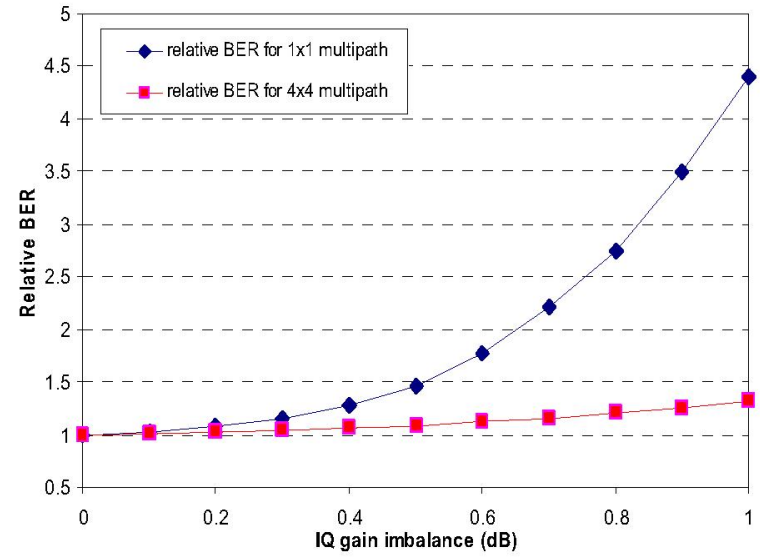

Fig. 6. Relative BER vs IQ phase imbalance in AWGN channel

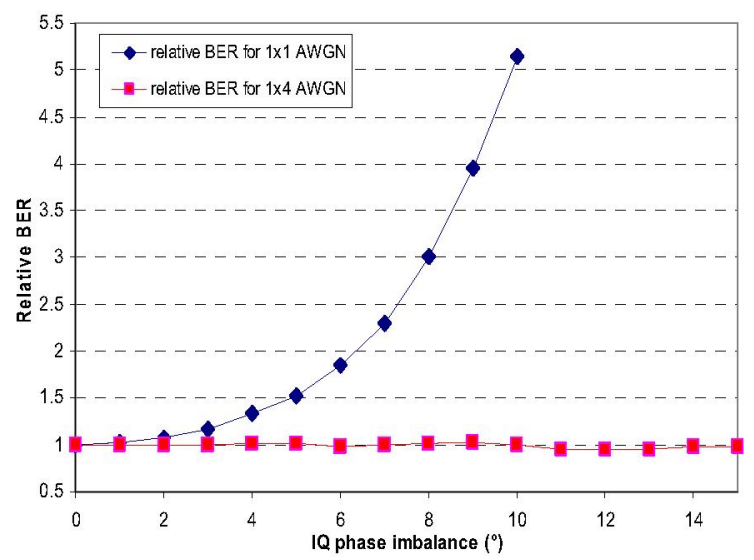

Fig. 7 Relative BER vs. IQ gain imbalance in multipath channel

receiver and a four antennas receiver in different channels and for gain or phase IQ imbalance, showing the potential of such a technique for joint fading/RF impairments mitigation.

\section{B. Influence of Power Amplifier with Nonlinear Gain Compression}

It's very interesting to study the effects of harmonic [6] distortion and intermodulation distortion. Indeed, a linear PA is needed in the case of non-constant envelope signals. The linearity of a PA is a key parameter as it is closely related to power consumption and to distortion, hence BER. All gain compression characteristics are modeled (with ADS software) using a polynomial expression up to the saturation. Fig. 8 shows characterization parameters for nonlinear elements. In Fig. 9, the effects of the 1-dB compression point and the intermodulation intercept point (IP) associated with the thirdorder products on BER performances (for $\mathrm{Eb} / \mathrm{No}=10 \mathrm{~dB}$ ) are reported for a multipath channel. Compared with the case of a AWGN channel (not reported), the SMI gain seems to be very interesting. Of course, measurements have to be performed to confirm these encouraging simulation results. 


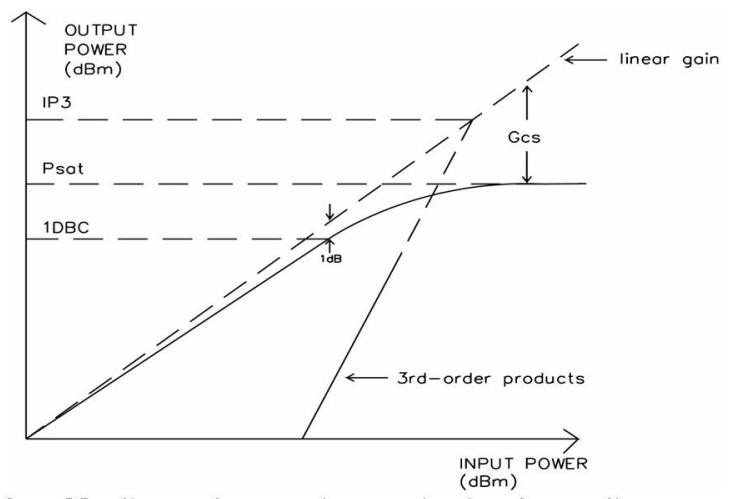

Fig. 8 Nonlinear element characterization for nonlinear models

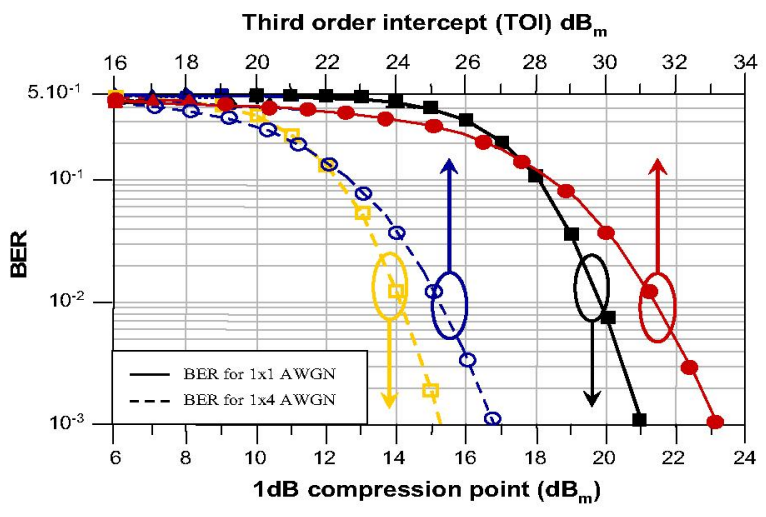

Fig. 9 BER versus TOI (upper axis) and $1 \mathrm{~dB}$ compression point (lower axis) in multipath channel (linear gain of PA is $10 \mathrm{~dB}$ and $\mathrm{Eb} / \mathrm{No}=10 \mathrm{~dB})$

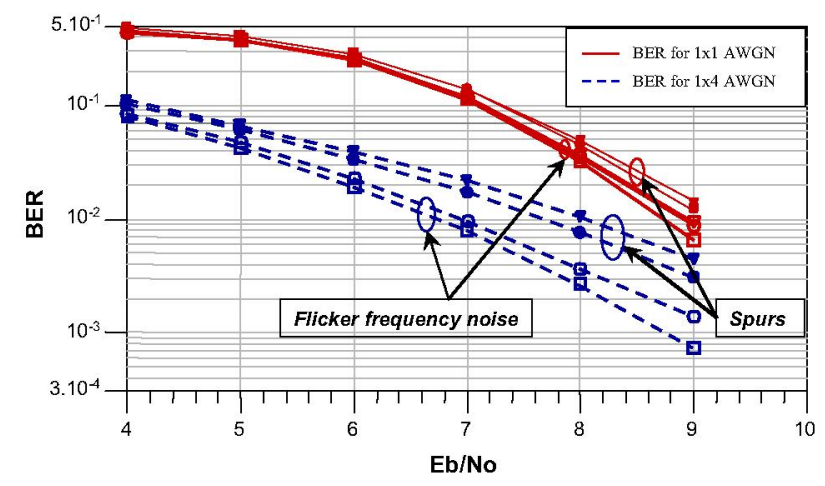

Fig. 10 Influence of phase noise (- $30 \mathrm{~dB} / \mathrm{dec})$ and PLL spurious on BER performances.

$$
\begin{aligned}
& -\square-:-90 \mathrm{dBc} / \mathrm{Hz} \text { at } 1 \mathrm{kHz} \text { offset } \\
& -\mathrm{O}--60 \mathrm{dBc} / \mathrm{Hz} \text { at } 1 \mathrm{kHz} \text { offset } \\
& -\boldsymbol{\nabla} \text { - : Spurs at } \pm 100 \mathrm{kHz} \text { is }-30 \mathrm{dBc} \text { (with phase noise : }-\mathrm{O} \text {-) } \\
& \text { - }- \text { - : Spurs at } \pm 100 \mathrm{kHz} \text { is }-60 \mathrm{dBc} \text { (with phase noise : }-\square \text {-) }
\end{aligned}
$$

\section{Influence of $O_{\text {scillators }}$ Phase Noise}

The same approach was used to study the influence of phase noise spectrum of local oscillators [7] and to evaluate the improvement of the traditional Sample Matrix Inversion.
Any phase noise model is easy to design and to implement in the ADS software. For instance, it is possible to analyse the impact of flicker frequency noise (a classical model for the phase noise in free running oscillators) and PLL spurious on BER curves vs. signal to noise ratio (spurious response or spur is a generic problem associated with fractional-N synthesizers [8]), see Fig. 10.

\section{CONCLUSION}

Today's wireless communications systems are built by multidisciplinary teams that include experts in signal processing, RF engineering and other disciplines. Our global system approach permits to easily develop, simulate and validate a wireless system even with a multi-antennas receiver structure (SIMO OFDM system). The efficiency of a complete connected solution based on Agilent Technologies tools, combining simulations and measurements under true operating conditions (correlated channels and antenna coupling) is clearly demonstrated. The IQ imbalance influence on a four antennas $802.11 \mathrm{~g}$ receiver has been presented, and the efficiency of the SMI algorithm for BER reduction has been clearly shown. We have also presented the compensation of phase noise thanks a SIMO receiver, and the impact of non-linear distortion of amplifiers on OFDM receiver performances.

\section{REFERENCES}

[1] P. Rykaczewski, et al, "Signal Path Optimization in SoftwareDefined Radio Systems" IEEE Trans. Mic. Th. And Tech., vol. 53 , No 3, 2005

[2] http://eesof.tm.agilent.com/products/ads_main.html

[3] I. J. Gupta, "SMI adaptive antenna arrays for weak interfering signals", IEEE Trans. Antennas and Propagat., vol. 34, pp $1237-1242,1986$

[4] J. Tubbax et al., "Joint Compensation of IQ Imbalance, Frequency Offset and Phase Noise in OFDM Receivers", European Trans. On Telecom., Special Issue on Multi-Carrier Spread-Spectrum, vol. 15, 2004

[5] M. Brandolini, et al, "Toward Multistandard Mobile Terminals - Fully Integrated Receivers Requirements and Architectures", IEEE Trans. Mic. Th. And Tech., vol. 53, No 3, 2005

[6] F.H. Gregorio and T.I. Laakso, "The performance of OFDMSDMA systems with power amplifier non-linearities" in Proc. 2005 Finnish Signal Processing Symposium, pp. 58-61, 2005

[7] S. Wu and Y. Bar-Ness, "Performance analysis on the effect of phase noise in OFDM systems," in Proc. IEEE Seventh International Symposium on Spread Spectrum Techniques and Applications, vol. 1, pp.133-138, 2002

[8] S. B. Razavi "Phase locking in high performance systems : From Devices to Architectures" publisher J. Wiley \& Sons Inc, 736 pages, 2003 